\title{
Selectivity on Ion Transport across Bilayer Lipid Membranes in the Presence of Gramicidin A
}

\author{
Shintaro Kubota, Shunsuke Ozaki, Jun Onishi, Kenji Kano, and Osamu Shirai ${ }^{\dagger}$ \\ Division of Applied Life Sciences, Graduate School of Agriculture, Kyoto University, \\ Sakyo, Kyoto 606-8502, Japan
}

\begin{abstract}
Ion transport from one aqueous (W1) to another (W2) across bilayer lipid membranes (BLM) containing gramicidin A (GA) was investigated by recording current fluctuations, when various alkali metal chlorides and potassium salts were used as supporting electrolytes. The magnitude of the single-channel current at a given membrane potential depended on not only the cationic species, but also on the anionic species, and then it decreased with an increase in the diameter of the anion when the diameter of the anion was larger than the pore size of the GA channel. The baseline of the recording current, however, increased with an increase in the diameter of the anion, and its height depended on the concentration of GA in the BLM. The results indicate that GA serves as not only a channel-forming compound, but also as a carrier compound in the BLM.
\end{abstract}

(Received September 18, 2008; Accepted September 29, 2008; Published February 10, 2009)

\section{Introduction}

Ion transport across biomembranes is associated with various biofunctions, such as aspiration, metabolism, photosynthesis, neurotransmission, etc. Ion transport from one aqueous phase (W1) to another (W2) across a bilayer lipid membrane (BLM), which is one of the simplest biomembrane models, has been generally utilized in order to interpret mechanisms of ion transport across biomembranes. ${ }^{1}$ Many studies have been performed on ion transport in the presence of ionophores, such as ion channels, ion channel-forming peptides, complex-forming agents and hydrophobic ions in BLM systems. ${ }^{2-8}$ It is generally recognized that ion transport currents are generated by only the transport of ions associated with their transporters. Gramicidin A (GA) is a linear pentadecapeptide isolated from Bacillus brevis, and exhibits antibiotic activity. ${ }^{9}$ However, it is believed that two molecules of GA form an ion channel within the BLM phase and small monovalent cations, such as alkali metal ions, permeate across the BLM easily. ${ }^{10}$ The influence of the counter anions on the transport of cations has not been considered. ${ }^{11-13}$ In this mechanism, however, the electroneutrality within the BLM is not considered.

It was pointed out that the magnitude of ion transport current across the BLM depended on the hydrophobicity of the counter anion coexisting with the objective cation. ${ }^{14-16}$ Watanabe et al. and Cohen reported that the permeability of the alkali metal cation across liposomal membranes containing GA depended on the species of the counter anions. ${ }^{17,18}$ The electrochemical behavior of a single channel of GA and the influence of the counter anion on the transport of a monovalent cation has not yet been investigated.

In the present work, the influence of ionic properties on the

† To whom correspondence should be addressed.

E-mail: shiraio@kais.kyoto-u.ac.jp facilitated ion transport across BLM containing GA was investigated by recording the current fluctuation. Taking into account the distribution of both the alkali metal cation and its counter anion and the relation between the pore size of the GA channel and the ionic diameter of the transport ions, we propose a new mechanism of ion transport.

\section{Experimental}

\section{Chemicals}

The lipids used to form BLM were lecithin (Wako Pure Chemical Ind., Ltd.; No. DPN4417) and cholesterol (Kanto Chemical Co., Inc.; No. 001G1157). Gramicidin A was purchased from Sigma Chemical Co. (St. Louis, MO; No. 017254/1). All other reagents were of reagent grade and used without further purification.

\section{Preparation of BLMs}

The electrochemical cell used for the experiments was essentially the same as that used in our previous work. ${ }^{14-16}$ The cell has two aqueous compartments separated by a tetrafluoroethylene resin sheet (Du Pont-Mitsui Fluorochemical Co., Ltd.) with a thickness of $0.2 \mathrm{~mm}$; the compartments were filled with $15 \mathrm{~mL}$ of an aqueous solution. The aqueous solution contained $20 \mathrm{mM}$ Bis-Tris buffer $(\mathrm{pH} 7.0)$ and $0.1 \mathrm{M} \mathrm{1:1}$ electrolyte salt. The BLM was obtained as a black lipid membrane by brushing a BLM-forming solution on a 1-mm diameter aperture created on a tetrafluoroethylene resin sheet. Gramicidin A was dissolved in ethanol at a concentration of about $10^{-3} \mathrm{M}$, and this solution served as a stock solution. An adequate volume of the ethanol solution was transferred to a $1-\mathrm{mL}$ flask. After ethanol was evaporated, BLM-forming solution was prepared by dissolving the residual GA, $10 \mathrm{mg}$ of lecithin and 5 $\mathrm{mg}$ of cholesterol into $n$-decane in the 1 -mL flask. The amount of GA in the BLM was estimated from the molar ratio of BLM 


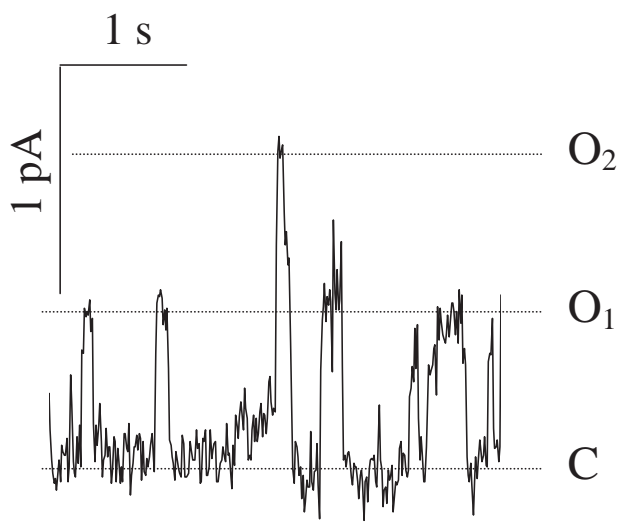

Fig. 1 Single-channel recording for GA in the presence of $0.1 \mathrm{M}$ $\mathrm{KCl}$. The applied potential was $+50 \mathrm{mV}$. Concentration of GA in the BLM-forming $n$-decane solution: $10^{-7} \mathrm{M}$. C indicates the closed state and $\mathrm{O}$ the open state.

components in the forming solution. Cholesterol was added to obtain stable BLMs. The formation of BLM was confirmed by capacitance measurements and microscopic observations of light reflection from the membrane surface.

\section{Single-channel recordings}

The ion transport current between W1 and W2 across BLM, $i_{\mathrm{W} 1-\mathrm{W} 2}$, was recorded by applying a constant potential difference between W1 and W2; $E_{\mathrm{W} 1-\mathrm{W} 2}$. $E_{\mathrm{W} 1-\mathrm{W} 2}$ was applied through two $\mathrm{Ag} \mid \mathrm{AgCl}$ electrodes, RE1 and RE2, soaked in $\mathrm{W} 1$ and $\mathrm{W} 2$, respectively, and is defined as the potential of RE1 referred to RE2. In this work, the positive current is defined as being due to the transports of positively charged species from W1 to W2 and those of negatively charged species from $\mathrm{W} 2$ to $\mathrm{W} 1 ; i_{\mathrm{W} 1-\mathrm{W} 2}$ was measured by two platinum wire electrodes. The current was sampled every $10^{-3} \mathrm{~s}$ and a low-pass filter with cut-off frequency of $10 \mathrm{~Hz}$ was used.

All electrochemical measurements were performed in a Faraday cage at room temperature using a four-electrode potentiostat Model HA-1010mM1A (Hokuto Denko Co.) and an A/D converter Model GL500 (Graphtec Co.).

\section{Results and Discussion}

Single-channel current of a gramicidin A-channel between two aqueous phases containing alkali metal chlorides

In the absence of GA in BLM, the conductance of BLM ranged from 10 to $30 \mathrm{pS}$. The mean area of BLM was about $3 \times 10^{-3} \mathrm{~cm}^{2}$. These BLMs were stable for a few hours, and no conductance fluctuation was observed in the range of $E_{\mathrm{W} 1-\mathrm{W} 2}$ from -120 to $+120 \mathrm{mV}$.

Figure 1 shows a typical trace of $i_{\mathrm{W} 1-\mathrm{W} 2}$ in the presence of GA. It was obtained for BLM containing GA between W1 and W2 containing $0.1 \mathrm{M} \mathrm{KCl}$ as a supporting electrolyte. In this case, the concentration of GA in the BLM-forming $n$-decane solution was $10^{-7} \mathrm{M}$, and the applied $E_{\mathrm{W} 1-\mathrm{W} 2}$ was $+120 \mathrm{mV}$. The dashed line (C) represents the closed state of the GA channel, and refers to the baseline. In the present case, a step-like current fluctuation was observed, and it related to the opening and closing of the GA channel. One or two channels were served $\left(\mathrm{O}_{1}\right.$ or $\left.\mathrm{O}_{2}\right)$. The single-channel current was evaluated as the difference between the mean value of the current flowing in the case of the opening state, $\mathrm{O}_{1}$, and that of the baseline current by

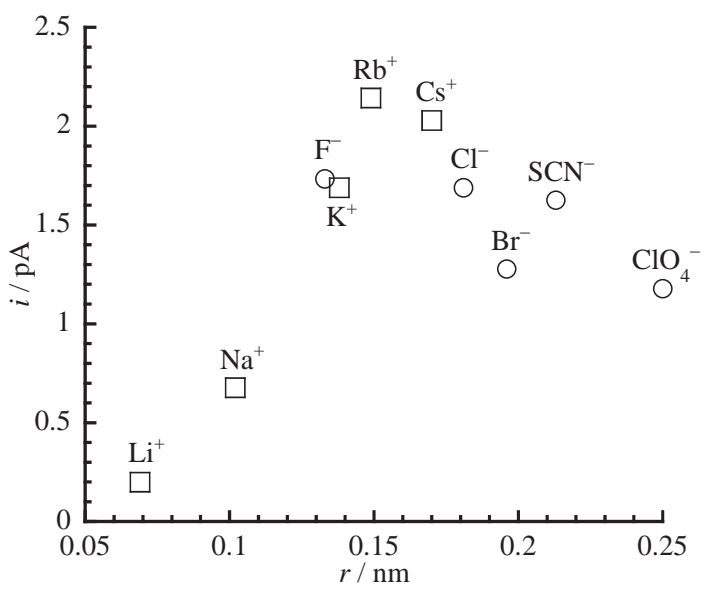

Fig. 2 Dependence of single-channel currents at $+120 \mathrm{mV}$ on the ionic radii of ions. All cations (open square) were used as chloride salts and all anions (open circle) were used as potassium salts dissolved in $20 \mathrm{mM}$ Bis-Tris buffer ( $\mathrm{pH}$ 7.0). Concentration of GA in the BLM-forming $n$-decane solution: $10^{-7} \mathrm{M}$.

Table 1 Ionic radius, $r$, of the ion and the standard molar Gibbs free energy of the hydration of the ion $^{24}$

\begin{tabular}{lccllc}
\hline Cation & $\begin{array}{c}\text { Ionic } \\
\text { radii, } \\
r / \mathrm{nm}\end{array}$ & $\begin{array}{c}\text { Hydration } \\
\text { energy, } \\
\Delta G_{\mathrm{hyd}}^{\circ} / \mathrm{kJ} \mathrm{mol}^{-1}\end{array}$ & Anion & $\begin{array}{c}\text { Ionic } \\
\text { radii, } \\
r / \mathrm{nm}\end{array}$ & $\begin{array}{c}\text { Hydration } \\
\text { energy, } \\
\Delta G_{\mathrm{hyd}}^{\circ} / \mathrm{kJ} \mathrm{mol}^{-1}\end{array}$ \\
\hline $\mathrm{Li}^{+}$ & 0.069 & -475 & $\mathrm{~F}^{-}$ & 0.133 & -465 \\
$\mathrm{Na}^{+}$ & 0.102 & -365 & $\mathrm{Cl}^{-}$ & 0.181 & -340 \\
$\mathrm{~K}^{+}$ & 0.138 & -295 & $\mathrm{Br}^{-}$ & 0.196 & -315 \\
$\mathrm{Rb}^{+}$ & 0.149 & -275 & $\mathrm{SCN}^{-}$ & 0.213 & -280 \\
$\mathrm{Cs}^{+}$ & 0.170 & -250 & $\mathrm{ClO}_{4}^{-}$ & 0.250 & -205 \\
$\mathrm{TMA}^{+}$ & 0.280 & -160 & & & \\
$\mathrm{TEA}^{+}$ & 0.337 & 0 & & & \\
$\mathrm{TBA}^{+}$ & 0.413 & - & & & \\
\hline
\end{tabular}

analyzing current-amplitude histograms of single-channel activities observed at each $E_{\mathrm{W} 1-\mathrm{W} 2}$. From the data obtained at $E_{\mathrm{W} 1-\mathrm{W} 2}$ ranging from +40 to $+120 \mathrm{mV}$, single-channel currents were practically proportional to $E_{\mathrm{W} 1-\mathrm{W} 2}$.

As shown in Fig. 2, the single-channel current depends on the cation species and the selectivity is similar to those reported by other authors. ${ }^{11-13,19,20}$ The single-channel current increased with an increase in the ionic diameter when the ionic diameter was smaller than about $0.30 \mathrm{~nm}$. It is thought that this trend was caused by stabilization of the cation within the pore of the GA channel due to the association of the cation with GA. ${ }^{13,19,20}$ On the other hand, several research groups reported that the GA channel was blocked by quarternary ammonium ions, such as tetramethylammonium ion $\left(\mathrm{TMA}^{+}\right)$, tetraethylammounium ion $\left(\mathrm{TEA}^{+}\right)$, tetrabutylammonium ion $\left(\mathrm{TBA}^{+}\right){ }^{13,21-23}$ Table 1 indicates the ionic radius and hydration energy of various ions..$^{24}$ Taking into account the fact that the radius of these quarternary ammonium ions was much larger than the pore size of the GA channel (i.d. $0.38 \mathrm{~nm}),{ }^{9,13,23}$ we can conclude that the GA channel exhibits the cation selectivity mentioned above on monovalent cations.

Single-channel current of gramicidin A-channel between two aqueous phases containing potassium salts

Figure 3(i) indicates the conventional ion-transport mechanism in the presence of GA channels. ${ }^{9,10}$ It was frequently mentioned 
(i)
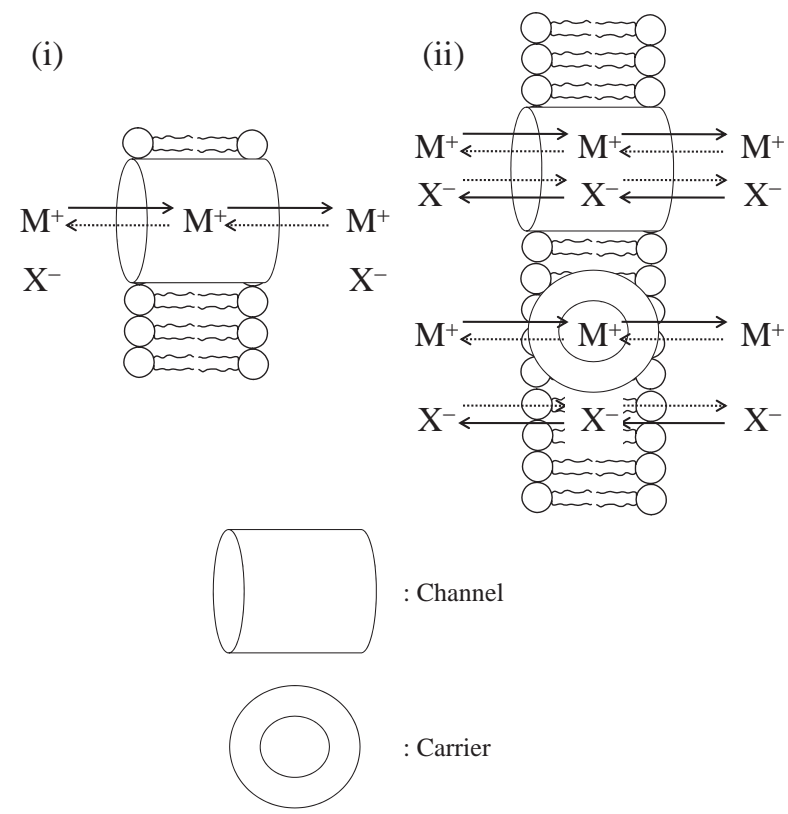

Fig. 3 Schematic model of ion-transport mechamisms in the presence of GA. $\mathrm{M}^{+}$, cation; $\mathrm{X}^{-}$, anion. (i) Conventional model and (ii) our proposed model.

that the GA channel served as a cation-selective transporter without any anion transport. On the other hand, some research groups reported on the dependence of counter anions on the permeability of electrolytes through the GA channels by the use of liposomal membranes. ${ }^{17,18}$ Then, we also revealed by cyclic voltammetry previously that the current density for the transport of $\mathrm{K}^{+}$across the BLM containing GA increased with an increase in the hydrophobicity of the counter anion. ${ }^{15}$ In the previous work, the current density at a given $E_{\mathrm{W} 1-\mathrm{W} 2}$ was relative to the square of the concentration of GA in the BLM when the ionic diameter of the counter anion was smaller than the pore size of the GA channel. This property was in agreement with that of the channel-transport model. ${ }^{13,23}$ However, the current density at a given $E_{\mathrm{W} 1-\mathrm{W} 2}$ was proportional to the concentration of GA in the BLM when the ionic diameter of the counter anion was larger than the pore size of the GA channel. Therefore, it was thought that the ion-transport current was attributable to the ion transport across the lipid layer site facilitated by GA as a carrier compound of $\mathrm{K}^{+} .{ }^{15}$

In the present work, the single-channel current of the GA channel between two aqueous phases containing various potassium salts was evaluated. The result indicated that the single-channel current gradually decreased with increasing the diameter of the counter anion, when the diameter of anionic species was larger than the inner diameter of the channel pore, as shown in Fig. 2. The single-channel current obtained in the case of $\mathrm{Cl}^{-}$was almost identical to that in the case of $\mathrm{F}^{-}$. It is contemplated that the magnitude of the ion-transport current may depend on the amounts of transferring ions, $\mathrm{K}^{+}$and the counter anion, in the BLM and on the diffusion coefficients of the ions. Therefore, we will discuss the ion transport of a single-charged cation, $\mathrm{M}^{+}$, and that of a single-charged anion (counter ion), $\mathrm{X}^{-}$, across the BLM in the presence of the same concentration of MX in W1 and W2, as presented using

$$
c_{\mathrm{i}}^{\mathrm{W} 1, \mathrm{o}}=c_{\mathrm{i}}^{\mathrm{W} 2, \mathrm{o}}=c_{\mathrm{i}}
$$

where $c_{\mathrm{i}}$ is the concentration of one ion (i) in $\mathrm{W} 1$ and $\mathrm{W} 2, c_{\mathrm{i}}{ }^{\mathrm{W} 1, \mathrm{o}}$ is the concentration of $i$ in the neighborhood of the W1|BLM interface in $\mathrm{W} 1$, and $c_{\mathrm{i}}{ }^{\mathrm{W} 2, \mathrm{o}}$ is the concentration of $\mathrm{i}$ in the neighborhood of the BLM|W2 interface in W2. Under the Goldman assumption, ${ }^{25,26}$ the flux of $\mathrm{i}, J_{\mathrm{i}}$, is given as

$$
J_{\mathrm{i}}=-\frac{D_{\mathrm{i}} z_{\mathrm{i}} F}{R T d} \Delta E\left\{\frac{c_{\mathrm{i}}^{\mathrm{W} 2, \text { in }} \exp \left(\frac{z_{\mathrm{i}} F}{R T} \Delta E\right)-c_{\mathrm{i}}^{\mathrm{W} 1, \text { in }}}{\exp \left(\frac{z_{\mathrm{i}} F}{R T} \Delta E\right)-1}\right\},
$$

where $D_{\mathrm{i}}$ is the diffusion coefficient of $\mathrm{i}$ in the BLM, $z_{\mathrm{i}}$ the charge of i, $F$ Faraday constant, $R$ the gas constant, $T$ the temperature in $\mathrm{K}, d$ the thickness of the BLM, $\Delta E$ potential difference between $\mathrm{W} 1$ and $\mathrm{W} 2, c_{\mathrm{i}}^{\mathrm{W} 1 \text {,in }}$ is the concentration of $\mathrm{i}$ in the neighborhood of the W1|BLM interface in the BLM, and $c_{\mathrm{i}}^{\mathrm{W} 2 \text {,in }}$ is the concentration of $\mathrm{i}$ in the neighborhood of the BLM|W2 interface in the BLM. The distribution coefficient of one ion (i), $\beta_{\mathrm{i}}$, is defined as

$$
\beta_{\mathrm{i}}=\frac{c_{\mathrm{i}}^{\mathrm{W} 1, \mathrm{in}}}{c_{\mathrm{i}}^{\mathrm{W} 1, \mathrm{o}}}=\frac{c_{\mathrm{i}}^{\mathrm{W} 2, \mathrm{in}}}{c_{\mathrm{i}}^{\mathrm{W} 2, \mathrm{o}}} .
$$

By using Eqs. (1) and (3), Eq. (2) can be rewritten as

$$
J_{\mathrm{i}}=-\frac{c^{\mathrm{o}} z_{\mathrm{i}} F}{R T d} \Delta E \beta_{\mathrm{i}} D_{\mathrm{i}}
$$

The current density of one ion (i), $j_{\mathrm{i}}$, is given by

$$
j_{\mathrm{i}}=-\frac{c_{\mathrm{i}}^{\mathrm{o}} z_{\mathrm{i}}^{2} F^{2}}{R T d} \Delta E \beta_{\mathrm{i}} D_{\mathrm{i}}
$$

There are two ion species, $\mathrm{M}^{+}$and $\mathrm{X}^{-}$, in the present ion transport system. Taking into account the electroneutrality within each phase, $\beta_{\mathrm{M}^{+}}$is equal to $\beta_{\mathrm{X}}$,

$$
\beta_{\mathrm{M}^{+}}=\beta_{\mathrm{X}^{-}}=\beta
$$

Therefore, the observed ion-transport current density, $j_{\text {total }}$, is equal to the sum of $j_{\mathrm{M}^{+}}$and $j_{\mathrm{X}}$,

$$
j_{\text {total }}=-\frac{c^{\circ} F^{2}}{R T d} \Delta E \beta\left(D_{\mathrm{M}^{+}}+D_{\mathrm{X}^{-}}\right) .
$$

Then, the distribution coefficient, $\beta$, is expressed as Eq. (8) based on the distribution of ions at an aqueouslorganic interface, ${ }^{27}$

$$
\ln \beta=-\frac{\Delta G_{\mathrm{tr}, \mathrm{M}^{+}}^{\circ}+\Delta G_{\mathrm{tr}, \mathrm{X}^{-}}^{\circ}}{2 R T}
$$

where $\Delta G_{\mathrm{tr}, \mathrm{i}}^{\circ}$ is the standard molar Gibbs energy of transfer of $\mathrm{i}$ from the aqueous phase to the BLM. The $\Delta G_{\mathrm{tr}, \mathrm{i}}^{\circ}$ value, however, is not evaluated exactly, because the BLM is too thin to measure the ion concentration. Therefore, we may utilize the hydration

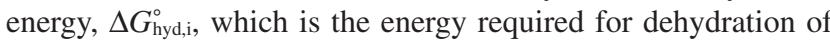
the ion as a measure of $\Delta G_{\mathrm{tr}, \mathrm{i}}^{\circ}$ by considering the fact that $\Delta G_{\mathrm{hyd}, \mathrm{i}}^{\circ}$ is proportional to $\Delta G_{\mathrm{tr}, \mathrm{i}}^{\circ}$ from W to organic solvents, such as nitrobenzene, 1,2-dichroloethane. ${ }^{28,29}$ Accordingly, Eq. (8) can be rewritten as

$$
\ln \beta=-\frac{1}{2 R T}\left\{\left(A_{+} \Delta G_{\mathrm{hyd}, \mathrm{M}^{+}}^{\circ}+A_{-} \Delta G_{\mathrm{hyd}, \mathrm{X}}^{\circ}\right)+\left(B_{+}+B_{-}\right)\right\} .
$$




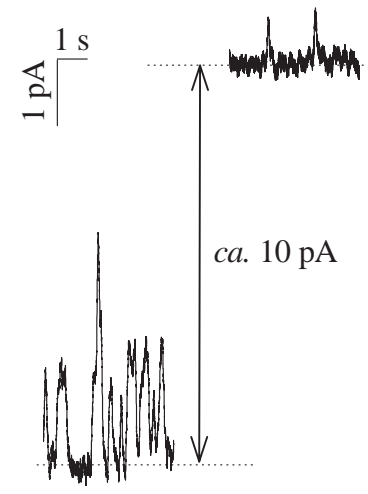

$O A$

Fig. 4 Single-channel recordings in the presence of GA. Supporting electrolyte in $\mathrm{W} 1$ and $\mathrm{W} 2: 0.1 \mathrm{M} \mathrm{KCl}$ (left) and $0.1 \mathrm{M}$ $\mathrm{KClO}_{4}$ (right).

Here, $\mathrm{A}_{+}, \mathrm{A}_{-}, \mathrm{B}_{+}$and $\mathrm{B}_{-}$are specific coefficients that are dependent on the solvent species. Equations (7) and (9) indicate that the magnitude of $j_{\text {total }}$ at a given $\Delta E$ depends on both the diffusion coefficients of not only the counter anion, but also the cation and the distribution coefficients, and is proportional to $\Delta E$. In addition, not only the counter anion, but also the alkali metal cation can hardly distribute from the aqueous phase to the BLM, because the counter anion cannot enter the channel pore when the ionic diameter is still larger than the pore size of the GA channel. In addition, the counter anion can hardly move within the channel pore. It is reasonable to suppose that a decrease in both the amount of the electrolytes within the channel pore and the diffusion coefficient through the channel pore causes a decrease in the magnitude of the single-channel current. Therefore, it seems reasonable to conclude that large anions, such as $\mathrm{ClO}_{4}^{-}$and $\mathrm{SCN}^{-}$, reduce the ion permeation of not only the counter anion, but also the alkali metal cation through the GA channel. In practice, the magnitude of the single-channel current and the open-time channel probability decreased, as shown in Fig. 4, when $\mathrm{KClO}_{4}$ was used instead of $\mathrm{KCl}$ as an electrolyte. Similarly, it seems reasonable to assume that $\mathrm{TMA}^{+}, \mathrm{TEA}^{+}$and $\mathrm{TBA}^{+}$reduced the ion permeation through the GA channel. When the ionic diameter is smaller than the pore size of the GA channel, it is expected that $\beta$ increases with an increase in the ionic diameter by considering $\Delta G_{\text {hyd,i }}^{\circ}$ and the stabilization of the cation by association with GA. Since it can be assumed that $j_{\text {total }}$ mainly depends on $\beta$ in this case, $j_{\text {total }}$ might increase with an increase in the ionic diameter. On the other hand, the baseline current rose by about $10 \mathrm{pA}$ when $\mathrm{KClO}_{4}$ or $\mathrm{KSCN}$ was used instead of $\mathrm{KCl}$ as a supporting electrolyte. The magnitude of the baseline shift was in proportion to the concentration of GA within the BLM. By considering the data reported previously, ${ }^{15}$ this result indicates that $\mathrm{ClO}_{4}{ }^{-}$and $\mathrm{SCN}^{-}$ facilitated the transport of $\mathrm{K}^{+}$across the lipid layer site by the addition of GA to the BLM. It is found from the result that GA served as a carrier compound of $\mathrm{K}^{+}$, as shown in Fig. 3(ii).

\section{Ion transport mechanism through gramicidin A-channel}

Figure 3(ii) indicates a new mechanism of the ion transport. It consists of two transportation routes. One is ion transport across the lipid bilayer site. The other is ion transport through the pore of the GA channel, which is related to the single-channel current.

The amounts of transport ions across the lipid layer site are related to the height of the current baseline, as mentioned above. It is clear that the amounts of transport ions depend on the Gibbs free energies of the electrolyte ions and on the complex formation energy between the alkali metal cation and the GA molecule, based on the ion transport mechanism reported in a previous work. ${ }^{15}$ Therefore, when an anion such as $\mathrm{ClO}_{4}{ }^{-}$and $\mathrm{SCN}^{-}$, of which diameter is larger than the pore size of the GA channel, is used as the supporting electrolyte, the height of the current baseline increased.

The magnitude of the single-channel current increased with increasing the diameter of the transport ion, when the diameter of cationic species was smaller than the inner diameter of the channel pore. It seems reasonable to assume that the selectivity for ion permeation results from both the hydrophobicity of the transport ion and stabilization due to the interaction between the cation and the GA molecules. The magnitude of the singlechannel current, however, decreased with an increase in the diameter of the transport ion, when the diameter of the transport ion, such as $\mathrm{TEA}^{+}, \mathrm{TBA}^{+}, \mathrm{ClO}_{4}^{-}, \mathrm{SCN}^{-}$, etc. was larger than the inner diameter of the channel pore. It is quite likely that the ion of which the diameter is larger than the diameter of the GA channel pore closes the channel gateway and hardly moves within the channel pore.

\section{Conclusions}

A new mechanism for ion transport across BLM in the presence of GA is proposed. The magnitude of the single-channel current at a given membrane potential depended on not only the cationic species, but also the anionic species. It decreased with an increase in the diameter of the anion when the diameter of the anion was larger than that of the GA channel. The baseline of the current recordings, however, increased with an increase in the diameter of the anion. The results indicate that the facilitated ion transport by GA consists of ion transport across the lipid bilayer site and that through the channel pore.

\section{Acknowledgements}

The authors thank Dr. Seiya Tsujimura, Graduate School of Agriculture, Kyoto University, for his helpful comments.

\section{References}

1. R. B. Gennis, "Biomembrane: Molecular Structure and Function", 1990, Springer-Verlag, New York.

2. H. T. Tien, "Bilayer Lipid Membranes", 1974, Marcel Dekker, New York.

3. B. H. Honig, W. L. Hubbell, and R. F. Flewelling, Annu. Rev. Biophys. Biophys. Chem., 1986, 15, 163.

4. H. T. Tien, Prog. Surf. Sci., 1985, 19, 169.

5. B. Hille, "Ion Channels of Excitable Membranes", 3rd ed., 2001, Sinauer Associates, Inc., Sunderland.

6. D. J. Aidley and P. R. Stanfield, "Ion Channels: Molecules in Action", 1996, Cambridge University Press, Cambridge.

7. E. Gouaux and R. MacKinnon, Science, 2005, 310, 1461.

8. T. W. Bell, Curr. Opin. Chem. Biol., 1998, $2,711$.

9. O. S. Andersen, R. E. Koeppe, and B. Roux, IEEE Trans. 
Nanobiosci., 2005, 4, 10.

10. P. Läuger, Angew. Chem., Int. Ed. Engl., 1985, 24, 905.

11. G. Eisenman, J. Sandblom, and E. Neher, Biophys. J., 1978, 22, 307.

12. G. A. Woolley and B. Wallace, J. Membr. Biol., 1992, 129, 109.

13. V. B. Myers and D. A. Haydon, Biochim. Biophys. Acta, 1972, 274, 313.

14. O. Shirai, Y. Yoshida, M. Matsui, K. Maeda, and S. Kihara, Bull. Chem. Soc. Jpn., 1996, 69, 3151.

15. O. Shirai, Y. Yoshida, S. Kihara, T. Ohnuki, A. Uehara, and H. Yamana, J. Electroanal. Chem., 2006, 595, 53.

16. O. Shirai, H. Yamana, T. Ohnuki, Y. Yoshida, and S. Kihara J. Electroanal. Chem., 2004, 570, 219.

17. S. Watanabe, S. Watanabe, and M. Seno, J. Membr. Sci. 1989, 44, 253.
18. B. E. Cohen, J. Membr. Biol., 1982, 68, 79.

19. O. S. Andersen, Biophys. J., 1983, 41, 119.

20. E. Neher, J. Sandblom, and G. Eisenman, J. Membr. Biol., 1978, 40, 97.

21. O. S. Andersen, Biophys. J., 1983, 41, 147.

22. K. Bokvist and J. Sandblom, J. Membr. Sci., 1992, 66, 157.

23. S. B. Hladky and D. A. Haydon, Biochim. Biophys. Acta, 1972, 274, 294.

24. Y. Marcus, J. Chem. Soc., Faraday Trans., 1991, 87, 2995.

25. D. E. Goldman, J. Gen. Physiol., 1943, 27, 37.

26. A. L. Hodgkin and B. Katz, J. Physiol., 1949, 108, 37.

27. Y. Yoshida, M. Matsui, O. Shirai, K. Maeda, and S. Kihara, Anal. Chim. Acta, 1998, 373, 213.

28. T. Osakai and K. Ebina, J. Phys. Chem. B, 1998, 102, 5691.

29. Y. Marcus, Biophys. Chem., 1994, 51, 111. 